\title{
Diagnosis of Maedi-Visna in Dairy Sheep
}

Created by: Aphrodite I. Kalogianni

Version received: 12 April 2020

check for updates

Maedi-visna in sheep is a chronic viral disease with worldwide distribution causing significant monetary losses in the sheep sector. Lack of effective treatment or vaccines, knowledge gaps regarding the epizootiology of the disease (e.g. significance of vertical and horizontal transmission) and the scarcity of protocols for the early and efficient diagnosis of maedi-visna infections are the major obstacles in the implementation of universally accepted control programs. The diagnostic methods currently used are based on the detection of either antibodies or the viral genome. In general, accuracy, sensitivity and specificity of the available serological and molecular assays vary and therefore, modifications on the diagnostic protocols on an evidential basis are necessary. Therefore, the combination of diagnostic assays and the development of effective strategies for mass implementation thereof are critical targets to achieve and constitute major challenges in maedi-visna research field.

Table of Content [Hide]

\section{Introduction}

Maedi-Visna (MV) (also known as ovine progressive pneumonia, OPP) is an incurable viral disease of sheep with very long incubation period that leads to life-long infection ${ }^{[1]}$. It is caused by non-oncogenic exogenous retroviruse ${ }^{2]}$, namely, maedi-visna virus (MVV) and caprine arthritis-encephalitis virus (CAEV), both belonging to a subgroup of viruses known as small ruminant lentiviruses (SRLVs). Its clinical manifestations are mainly pneumonia and mastitis, and secondarily, arthritis and encephalitis ${ }^{[3]}$. It causes substantial economic losses associated with involuntary culling or death of infected animals and reduced productivity, challenging the sustainability of dairy sheep farms. As there is no treatment against MV and all efforts for the development of vaccines did not produce any satisfactory results ${ }^{[4]}$, the control of the disease and the reduction of its prevalence have been achieved to some degree through controlled eradication programs aiming at diagnosis of the infected animals at an early stage.

Hence, early and efficient diagnosis of MV is a critical parameter for the control and eradication of the disease. The diagnosis of SRLVs infections is based on the detection of either antibodies against the virus or the viral genome. Current control and eradication programs are based on serological tests (mainly Enzyme-linked Immunosorbent Assay-ELISAs) to detect antibodies against the virus ${ }^{[5]}$. Therefore, only the seropositive animals are considered infected and subsequently removed from the flock. This is a major drawback for the eradication of the disease as the immune response to the disease (seroconversion) requires a long period of time and thereby, many of the infected animals remain undiagnosed carriers of the virus ${ }^{[6][7]}$. The available assays for the detection of antibodies are the Agar Gel ImmunoDiffusion (AGID) test, RadiolmmunoPrecipitation Assay (RIPA), Western Blotting (WB) and ELISA as described below.

\section{Agar gel immunodiffusion test (AGID test)}

AGID test is commonly used as a diagnostic tool in MV control programs due to its simplicity. It is a highly specific diagnostic method but less sensitive than ELISA ${ }^{[4][8][9]}$. For this reason, it is supplementary used for the confirmation of the ELISA test ${ }^{[10]}$. The results are qualitative and subjective as they are visually assessed (lines in agar . $^{[3][9][11]}$. AGID cannot be used for the detection of antibodies in milk ${ }^{[10]}$.

\section{Radioimmunoprecipitation assay (RIPA)}

RIPA is as old as WB and both of them are considered as the reference standards. They have similar sensitivity and are mainly used as confirmatory assays ${ }^{[9][11]}$. RIPA is not frequently used due to its high cost and its difficult application, as radiolabeling of the virus from cell cultures is required before running the test ${ }^{[9]}$. RIPA's results are imprinted on film and rely on antibody-mediated immunoprecipitation of ${ }^{35}$ S-labelled virus. The use of radio-labelled virus makes RIPA a more sensitive method than AGID ${ }^{[9]}$. 


\section{Western blot (WB)}

WB is a confirmatory laboratory test which has been used to detect antibodies in serum that recognize viral proteins. It is based on protein gel electrophoresis of cell lysates (cells infected in cultures with MVV), subsequent transfer onto either Nitrocellulose or Polyvinylidene difluoride (PVDF) membranes, and immunostaining utilizing whole animal sera as the primary source of antibodies. In the case of MVV, it is primarily used to detect the presence of antisurface envelope glycoprotein gp135SU, the presence of antitransmembrane protein gp46TM, and the presence of anticapsid CA antibodies in prospective sera ${ }^{[0][10][11]}$. In general, WB is more sensitive than the ELISAs but more cumbersome and with lower throughput ${ }^{[4][9]}$. Cross-reactivity with non-specific cellular proteins is also a problem. This is the reason that WB is only used for confirmation of ELISA and AGID test results.

\section{ELISA}

ELISA is the most commonly used test in population screening and for the surveillance of MV. It detects antiviral antibodies in milk, serum or semen and allows quantitative interpretation ${ }^{[12]}$, with satisfactory sensitivity and specificity, indicating the occurrence of infection and seroconversion at some point ${ }^{[4][10]}$. Seropositivity is not necessarily followed by clinical disease and a seronegative animal cannot be safely considered to be free of infection. In many cases, seroconversion requires several months even in early-infected animals (e.g., lambs infected via colostrum consumption) ${ }^{[7]}$. Moreover, the antigenic heterogeneity of SRLVs strains (especially among different subtypes, like the CAEV-like and the MVV-like) may limit the diagnostic performance of currently available ELISA ${ }^{[13]}$. Antibody titers present remarkable variations during animal's life and in some cases they are undetectable using an ELISA, which renders the test unreliable for a definite diagnosis ${ }^{[7][9][14][15]}$.

Four ELISA assays have been validated against RIPA, AGID or WB assays and found to present sensitivity and specificity greater than $95 \%$ for the detection of anti-MVV antibodies in serum. Two of them are non-competitive antibody detection ELISAs (nELISAs) and two are competitive inhibition ELISAs (cELISAs) ${ }^{[9]}$. In the first nELISA, whole-virus of Canadian CAEV strain serves as the coated antigen on plates (CAEV/MVV kit, IDEXX Switzerland AG, Liebefeld, Bern, Switzerland), whereas the only nELISA assay validated by the OIE is based on an assay in which recombinant CA and TM peptides from the MVV strain EV1 serve as the coated antigen (Elitest-MVV, HYPHEN Biomed, Neuville-sur-Oise, France and Pourquier $)^{[7][9]}$. Concerning the two cELISAs, the first one is an assay using anti-MVV, ZZV 1050 CA monoclonal antibodies and the second and more recent one is the CAEV-63 cELISA which uses two anti-CAEV-63 SU monoclonal antibodies (cELISA CAEV of VMRD Inc. Pullman, WA). These monoclonal antibodies compete with serum antibodies against binding sites on coated antigens in the plates. Except from the aforementioned tests which have been sufficiently validated, there are several other ELISA tests, like a recently developed ELISA including gag and env proteins from genotype A, B and $E$ which serve as the coated antigen onto the plates (IN3 diagnostic, Eradikit $\Theta$ SRLV indirect ELISA for Small Ruminant Lentiviruses). However, they need to be further validated ${ }^{[7]}$. As described above, in ELISAs, the coated antigens can be whole virus, recombinant proteins or synthetic peptides ${ }^{[10]}$. cELISAs present higher sensitivity than $\mathrm{nELISAs}$, but it is likely to be associated with reduced specificity, due to the use of undiluted sera ${ }^{[9]}$; moreover, they demand the use of monoclonal antibodies, unlike nELISAs ${ }^{[9]}$.

The validity of ELISA testing depends on the type of the host immune response. It has been found that increased antibody titers is the norm in the histiocytic pattern of immunological response, while low titers or negative results are observed in the case of lymphocytic immunological response ${ }^{[16]}$. This finding underpins the limitations of the implemented control programs using serological tests, indicating the critical role of undiagnosed, infected sheep in the epizootiology of MV. In addition, the current ELISA tests do not aid in identifying the circulating strain with high certainty. ELISA tests need to be strain-specific to be efficiently implemented in an area, a flock or specific animals ${ }^{[15]}$, otherwise, failure in antibody detection is likely to occur and the possibility of misdiagnosis increases.

\section{Detection of proviral DNA by Polymerase Chain Reaction (PCR)}

PCR can directly detect proviral DNA in fluids and tissues across the animal body (lungs, milk, peripheral blood, mammary gland, synovial membranes etc.). The most reliable cells for the detection of virus are the peripheral blood mononuclear cells ${ }^{[9][10]}$. The most significant advantage of PCR is its ability to detect infection before seroconversion. However, PCR is not a reference method and in practice is suggested to be combined with serological testing to

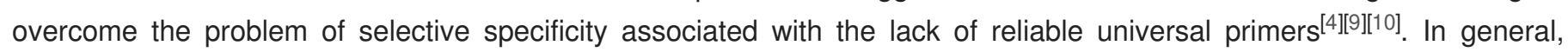


cELISAs are more sensitive and nELISAs and AGID are more specific than PCR, due to the non-generic applicability of the primers which are currently available ${ }^{[9]}$. PCR nested methods and Real-Time-PCR (RT-PCR) increase the sensitivity and specificity of the method, however, their use is less frequent ${ }^{[9][10]}$. In particular, RT-PCR is an efficient method for the detection and quantification of viral load, but its sophisticated designation and implementation set limitations upon its use $^{79}$. Nevertheless, several RT-PCR assays have been designed with various results regarding the efficiency and the quantification capabilities. Among them a RT-PCR assay for the gag gene of the EV1 strain is currently available and commonly used, but the continuous mutations of the virus seem to question its effectiveness in some cases ${ }^{[17]}$.

The development of primers for the different strains of MVV will improve the applicability and the value of PCR assays $^{[4][9][10]}$. Bulk milk testing using specific PCR assays need to be further exploited for the reduction of the cost, the time and effort required for blood sampling and analyses of individual animals ${ }^{[18]}$.

\section{Cell cultures}

The isolation of MVV can be achieved after the co-cultivation of animal leukocytes with sheep choroid plexus cells. The observed cytopathic effect (syncytia and refractile stellate cells with dendritic processes) is the evidence of MVV infection, which can be further confirmed with electronic microscopy ${ }^{[19]}$. This diagnostic method cannot be applied in a routine basis due to its cost, complexity and demand in labour and specialized equipment. Cell cultures are mainly used as a verification tool of other diagnostic methods or for research purpose. However, co-cultivation techniques hold promise in aiding very early identification of infected animals when only a small fraction of circulating immature macrophages are infected.

The development of a universally accepted and verified protocol for the early and definitive diagnosis of MV is crucial for the suggestion of prevention and eradication programs at national and international level and thereby, constitutes a challenging research field.

\section{References}

1. G.F. De Boer; Zwoegerziekte virus, the causative agent for progressive interstitial pneumonia (maedi) and meningoleucoencephalitis (visna) in sheep. Research in Veterinary Science 1975, 18, 15-25, 10.1016/s0034-5288(18)33625-7.

2. Pálsson, P.A. Maedi-Visna and Related Diseases. Developments in Veterinary Virology; Pétursson, G., HoffJørgensen, R., Eds.; Kluwer Academic Publishers: Norwell, MA, USA, 1990; pp. 3-74.

3. Otto Christian Straub; Maedi-Visna virus infection in sheep. History and present knowledge.Comparative Immunology, Microbiology and Infectious Diseases 2004, 27, 1-5, 10.1016/s0147-9571(02)00078-4.

4. Ernst Peterhans; Tim Greenland; Juan Badiola; Gordon Harkiss; Giuseppe Bertoni; Beatriz Amorena; Muriel Eliaszewicz; Ramón Juste; Renate Kraßnig; Jean-Pierre Lafont; et al.Patrick LenihanGudmundur PéturssonGeoff PritchardJohn ThorleyChristian VituJean-Francois MornexMichel Pepin Routes of transmission and consequences of small ruminant lentiviruses (SRLVs) infection and eradication schemes. Veterinary Research 2004, 35, 257-274, 10.1051/vetres:2004014.

5. Jarosław Kaba; Michał Czopowicz; Martin Ganter; Mariusz Nowicki; Lucjan Witkowski; Dorota Nowicka; Olga SzaluśJordanow; Risk factors associated with seropositivity to small ruminant lentiviruses in goat herds. Research in Veterinary Science 2013, 94, 225-227, 10.1016/j.rvsc.2012.09.018.

6. J. Brinkhof; C. Van Maanen; Evaluation of Five Enzyme-Linked Immunosorbent Assays and an Agar Gel Immunodiffusion Test for Detection of Antibodies to Small Ruminant Lentiviruses. Clinical and Vaccine Immunology 2007, 14, 1210-1214, 10.1128/cvi.00282-06.

7. Hugo Ramírez; Ramsés Reina; Beatriz Amorena; Damián Andrés; Humberto A. Martínez; Small Ruminant Lentiviruses: Genetic Variability, Tropism and Diagnosis. Viruses 2013, 5, 1175-1207, 10.3390/v5041175.

8. Esmeralda Minguijón; Ramsés Reina; M. Pérez; Laura Polledo; M. Villoria; H. Ramírez; Iratxe Leginagoikoa; J.J. Badiola; Juan Francisco García Martín; D.F. De Andres-Cara; et al.L. LujánB. AmorenaRamón Juste Small ruminant lentivirus infections and diseases. Veterinary Microbiology 2015, 181, 75-89, 10.1016/j.vetmic.2015.08.007.

9. L. M. Herrmann-Hoesing; Diagnostic assays used to control small ruminant lentiviruses...Journal of Veterinary Diagnostic Investigation 2010, 22, 843-855, 10.1177/104063871002200602.

10. Ramsés Reina; Eduardo Berriatua; L. Luján; Ramón Juste; Antonio Sánchez; D.F. De Andres-Cara; Beatriz Amorena; Prevention strategies against small ruminant lentiviruses: An update. The Veterinary Journal2009, 182, 31-37, 
10.1016/j.tvjl.2008.05.008.

11. D. De Andrés; D. Klein; N.J. Watt; E. Berriatua; Sigurbjörg Torsteinsdóttir; B.A. Blacklaws; G.D. Harkiss; Diagnostic tests for small ruminant lentiviruses. Veterinary Microbiology 2005, 107, 49-62, 10.1016/j.vetmic.2005.01.012.

12. Rodolphe Michiels; Eva Van Mael; Christian Quinet; Nadjah Adjadj; Ann Brigitte Cay; Nick De Regge; Comparative Analysis of Different Serological and Molecular Tests for the Detection of Small Ruminant Lentiviruses (SRLVs) in Belgian Sheep and Goats.. Viruses 2018, 10, 696, 10.3390/v10120696.

13. Daniela Lacerenza; Monica Giammarioli; Elena Grego; Carla Marini; Margherita Profiti; Domenico Rutili; Sergio Rosati; Antibody response in sheep experimentally infected with different small ruminant lentivirus genotypes. Veterinary Immunology and Immunopathology 2006, 112, 264-271, 10.1016/j.vetimm.2006.03.016.

14. D. De Andrés; D. Klein; N.J. Watt; E. Berriatua; Sigurbjörg Torsteinsdóttir; B.A. Blacklaws; G.D. Harkiss; Diagnostic tests for small ruminant lentiviruses. Veterinary Microbiology 2005, 107, 49-62, 10.1016/j.vetmic.2005.01.012.

15. X. De Andrés; H. Ramirez; Luigi Bertolotti; Beatriz San Román; I. Glaria; H. Crespo; Paula Jáuregui; Esmeralda Minguijón; Ramón Juste; Iratxe Leginagoikoa; et al.M. PérezL. LujánJ.J. BadiolaL. PolledoJ.F. García-MarínJosé I. Riezu-BojF. Borrás-CuestaD. De AndrésSergio RosatiRamsés ReinaBeatriz Amorena An insight into a combination of ELISA strategies to diagnose small ruminant lentivirus infections. Veterinary Immunology and Immunopathology 2013, 152, 277-288, 10.1016/j.vetimm.2012.12.017.

16. E. Gayo; L. Polledo; S. Preziuso; G. Rossi; Ana Balseiro; Claudia Perez-Martinez; M. José García Iglesias; J.F. García Marín; Serological ELISA results are conditioned by individual immune response in ovine maedi visna. Small Ruminant Research 2017, 157, 27-31, 10.1016/j.smallrumres.2017.10.008.

17. Maria Luisa Carrozza; Maurizio Mazzei; Patrizia Bandecchi; Christophe Fraisier; Marta Pérez; Marie Suzan-Monti; Damián De Andrés; Beatriz Amorena; Sergio Rosati; Valgerdur Andrésdóttir; et al.L. LujánMichel PepinBarbara BlacklawsFrancesco TolariGordon D. Harkiss Development and comparison of strain specific gag and pol real-time PCR assays for the detection of Visna/maedi virus. Journal of Virological Methods2010, 165, 161-167, 10.1016/j.jviromet.2010.01.013.

18. J. Brinkhof; D.J. Houwers; L. Moll; D. Dercksen; C. Van Maanen; Diagnostic performance of ELISA and PCR in identifying SRLV-infected sheep and goats using serum, plasma and milk samples and in early detection of infection in dairy flocks through bulk milk testing. Veterinary Microbiology 2010, 142, 193-198, 10.1016/j.vetmic.2009.09.060.

19. OIE. Chapter 3.7.2/3 Caprine Arthritis-Encephalitis \& Maedi-Visna. In: Manual of Diagnostic Tests and Vaccines for Terrestrial Animals 2019.

\section{Keywords}

maedi-visna; small ruminant lentivirus; diagnosis; dairy sheep (C) 2020 by the author(s). Distribute under aCreative Commans CC BY license 